\title{
Dinoflagelados em diversos habitats e hidroperíodos na zona costeira do sul do Brasil
}

\author{
Luciana de Souza Cardoso ${ }^{1,3}$ e Lezilda Carvalho Torgan ${ }^{2}$
}

\author{
Recebido em 5/05/2005. Aceito em 27/10/2006
}

\begin{abstract}
RESUMO - (Dinoflagelados em diversos habitats e hidroperíodos na zona costeira do sul do Brasil). Informações sobre a riqueza, densidade, diversidade e distribuição de dinoflagelados em habitats aquáticos (lagoas abertas e fechadas, canais, açudes e áreas úmidas), em relação aos períodos de águas altas e baixas, são apresentadas. O estudo baseou-se em amostragens realizadas em 23 pontos, localizados nas margens leste (área da Lagoa do Casamento) e oeste (área do Butiazal de Tapes) da laguna dos Patos, no ano de 2003. Foram identificados 11 táxons, cuja distribuição teve maior homogeneidade no período de águas altas. Durinskia baltica (Levander) Carty \& Cox foi o único táxon cuja distribuição não se alterou pelo hidroperíodo. Peridinium gatunense Nygaard foi uma espécie indicadora de águas altas e P. umbonatum Stein foi indicadora de habitats associados à área do Butiazal de Tapes. Esta área mostrou ter mais alta diversidade de Dinophyceae em relação à área da Lagoa do Casamento, independente do hidroperíodo, refletindo a maior diversidade e especificidade de habitats aquáticos. O tipo de habitat influenciou significativamente $(\mathrm{p}<0,01)$ a riqueza, densidade e diversidade de dinoflagelados, e a densidade foi significativamente $(\mathrm{p}<0,01)$ influenciada pelo hidroperíodo. A análise de agrupamento mostrou que $\mathrm{pH}$ e temperatura da água foram as variáveis abióticas que mais influências tiveram sobre a estrutura da comunidade de dinoflagelados neste estudo.
\end{abstract}

Palavras-chave: Dinophyceae, ecologia, água doce, zona subtropical

\begin{abstract}
Dinoflagellates in different habitats and hydroperiods on the coast of southern Brazil) Data on richness, density, diversity and distribution of dinoflagellates in aquatic habitats (open and closed lagoons, channels, reservoirs and wetlands) during high and low water periods are presented. The study was based on 23 point samples, located on the eastern (Lagoa do Casamento area) and western (Butiazal de Tapes area) shores of Laguna dos Patos, in 2003. Eleven taxa were identified; taxon distribution was more homogeneous during the high-water period. Durinskia baltica (Levander) Carty \& Cox was the only taxon whose distribution was the same for both hydroperiods. Peridinium gatunense Nygaard was a high-water indicator species and P. umbonatum Stein was an indicator of habitats associated with the Butiazal de Tapes area. This area had higher Dinophyceae diversity than the Lagoa do Casamento area, regardless of hydroperiod, due to the higher diversity and specificity of aquatic habitats. Habitat type significantly $(\mathrm{p}<0.01)$ influenced richness, density and diversity of dinoflagellates, and density was significantly influenced $(\mathrm{p}<0.01)$ by hydroperiod. Cluster analysis showed that $\mathrm{pH}$ and water temperature were the abiotic variables that had greater influence on the dinoflagellate populations in this study.
\end{abstract}

Key words: Dinophyceae, ecology, freshwater, subtropical zone

\section{Introdução}

Dinoflagelados de água doce compreendem 2.000 espécies atuais, pertencentes a aproximadamente 130 gêneros (Taylor 1987). Apesar da importância de Dinophyta em muitas águas doces, muito pouco é conhecido sobre os fatores que influenciam sua ocorrência (Grigorszky et al. 2003a). São organismos unicelulares com ciclo de vida que permite habitar, alternativamente, o plâncton (como células vegetativas móveis) e o bento (como cistos imóveis). Os dinoflagelados mais bem conhecidos ecologicamente são as espécies de Peridinium e Ceratium (Pollingher 1988).

Ceratium, Peridinium e Peridiniopsis são aparentemente cosmopolitas; preferem águas duras com alta concentração de cálcio e toleram uma ampla variação de condições ambientais. Muitos dinoflagelados são encontrados em corpos d'água bem oxigenados, pois evitam sistemas eutróficos que sofrem depreciação periódica de oxigênio. Por esta razão eles não são encontrados em tanques supridos com água

\footnotetext{
1 Universidade Federal do Rio Grande do Sul, Instituto de Biociências, Departamento de Botânica, Av. Bento Gonçalves 9500, Prédio 43433, Bairro Agronomia, 91501-970 Porto Alegre, RS, Brasil

2 Museu de Ciências Naturais, Fundação Zoobotânica do Rio Grande do Sul, Rua Dr. Salvador França 1427, Bairro Jardim Botânico, $90690-000$ Porto Alegre, RS, Brasil (torgan@cpovo.net)

3 Autor para correspondência: luciana.cardoso@ufrgs.br
} 
residuária (Pollingher 1988). Entretanto, relações entre a importância de táxons de Dinophyta e estado trófico têm sido controvérsias (Grigorszky et al. 2003a). Em regiões subtropicais, luz não é um fator limitante, pois está disponível ao longo do ano. Nestas regiões, a sucessão sazonal é fortemente influenciada por ventos e chuvas (Pollingher 1986).

O conhecimento sobre a diversidade de dinoflagelados de águas continentais no Brasil é limitado (Rocha 2000). No Rio Grande do Sul, apenas oito táxons foram registrados em águas dulciaquícolas (Torgan et al. 2003). Rosa et al. (1987) mencionaram a ocorrência de Peridinium gatunense Nygaard em açudes da região do curso inferior do rio Jacuí; Franceschini (1992) a presença de Peridinium volzii Lemmermann e Peridiniopsis oculatum (Stein) Bourrelly no lago Guaíba e lagos artificiais de Porto Alegre; Garcia \& Vélez (1995) citam Peridinium umbonatum Stein para lagoa costeira de Emboaba; e Torgan et al. (2003) registrou Gymnodinium aeruginosum Stein, G. incoloratum Conrad \& Kufferath, G. latum Skuja e Peridinium achromaticum Levander para a laguna dos Patos. O conhecimento da variação de atributos da comunidade em distintos habitats aquáticos da Planície Costeira era inexistente.

O presente trabalho teve por objetivo avaliar a riqueza, diversidade, distribuição e variação da densidade de dinoflagelados, levando em consideração os distintos habitats aquáticos do litoral médio do Rio Grande do Sul e o hidroperíodo, bem como identificar espécies indicadoras desta variação espacial e temporal.

\section{Material e métodos}

O estudo baseou-se em amostragens realizadas em habitats aquáticos localizados nas margens leste (área da Lagoa do Casamento) e oeste (área do Butiazal de Tapes) da laguna dos Patos, entre as coordenadas $30^{\circ} 40^{\prime} \mathrm{S}-30^{\circ} 10^{\prime} \mathrm{S}$ e $50^{\circ} 30^{\prime} \mathrm{W}-51^{\circ} 30^{\prime} \mathrm{W}$ (Fig. 1). Foram selecionados 23 pontos de amostragem (alguns locais com dois pontos de amostragem A e B). $\mathrm{Na}$ área da Lagoa do Casamento (LC), os tipos de habitats selecionados foram três lagoas abertas (P3, P4, P7-P9, P11-P12), dois banhados (P1-P2, P5-P6) e um canal (P10). Na área do Butiazal de Tapes, os tipos de habitats selecionados foram quatro lagoas fechadas (P13-P14, P16-P17, P19, P21), um açude (P15) e dois banhados (P18, P20). Estes ambientes foram amostrados, no turno da manhã, durante o período de águas altas (nível de água médio de 1,43 m), na estação de outono (maio e junho/2003), e período de águas baixas (nível de água médio de 1,07 m), na estação de primavera (outubro a dezembro/2003).

As amostragens em zonas pelágicas (em nove pontos, Tab. 1) foram realizadas com o uso de motobomba (Sthil P835), onde um volume de 200 ou $300 \mathrm{~L}$ foi concentrado em rede com malha de $25 \mu \mathrm{m}$. As amostras em zonas litorâneas (em 15 pontos, Tab. 1) foram obtidas com rede de arraste superficial e através de espremido de macrófitas aquáticas. $\mathrm{O}$ material foi fixado com formol $4 \%$.

As espécies foram identificadas de acordo com Grigorszky et al. (2003b), Popovsky (1970; 1983) Popovsky \& Pfiester (1986; 1990) e Thompson (1950). Alguns organismos foram identificados em nível genérico e outros em conferatur, devido a carência de estudos taxonômicos sobre Dinophyceae em águas continentais brasileiras.

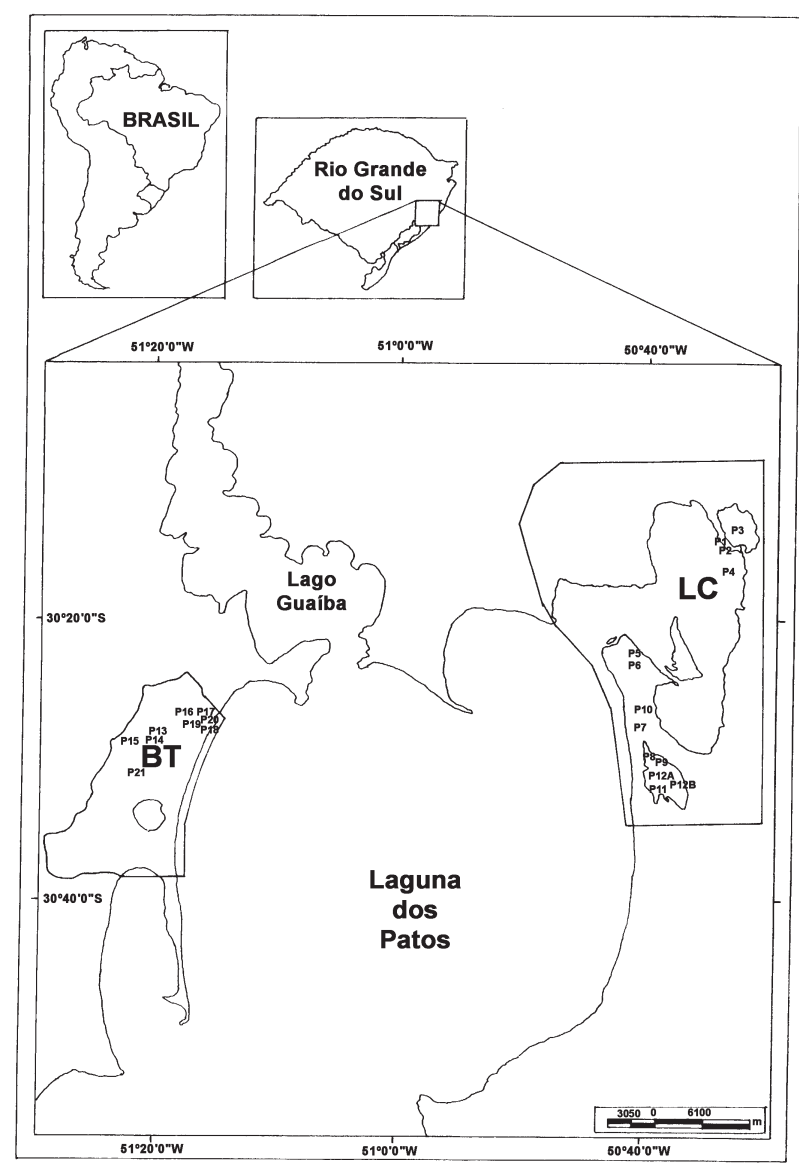

Figura 1. Localização dos pontos de amostragem nas respectivas áreas de estudo (Lagoa do Casamento - LC e Butiazal de Tapes $\mathrm{BT})$, na região do litoral médio do Rio Grande do Sul. 
Tabela 1. Dinophyceae ocorrentes nos habitats aquáticos associados à Lagoa do Casamento e ao Butiazal de Tapes, durante o hidroperíodo de águas altas $(\mathrm{A})$ e baixas $(\mathrm{B})(\mathrm{P}=$ pelágica; $\mathrm{L}=$ litorânea).

Áreas

\begin{tabular}{|c|c|c|c|c|c|c|c|c|c|c|c|c|c|}
\hline \multicolumn{7}{|c|}{ Lagoa do Casamento } & \multicolumn{7}{|c|}{ Butiazal de Tapes } \\
\hline & $\hat{2}$ & I & $\begin{array}{l}0 \\
2 \\
n \\
n\end{array}$ & $\begin{array}{l}\stackrel{2}{2} \\
\frac{1}{2}\end{array}$ & $\stackrel{\circ}{a}$ & $\frac{\vec{a}}{a}$ & $\begin{array}{l}\frac{m}{a} \\
\stackrel{1}{a} \\
\frac{a}{2}\end{array}$ & $\frac{n}{a}$ & $\frac{a}{a}$ & $\frac{\infty}{a}$ & $\frac{a}{2}$ & ָิ & $\bar{\Omega}$ \\
\hline
\end{tabular}

Pontos de amostragem

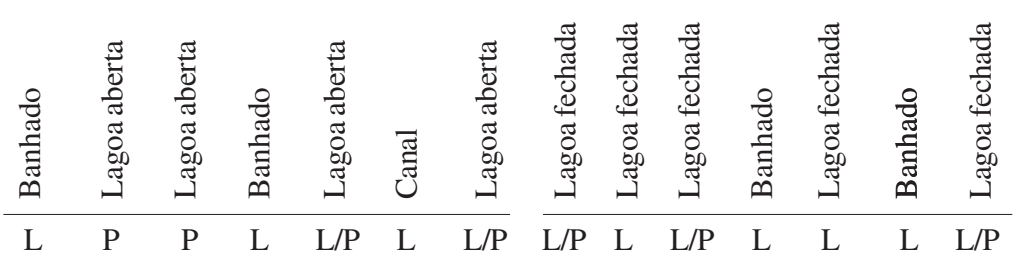

Habitat aquático

Zonas

\begin{tabular}{|c|c|c|c|c|c|c|c|c|c|c|c|c|}
\hline \multirow{9}{*}{ A } & \multicolumn{12}{|c|}{ Hidroperíodo } \\
\hline & $\mathrm{A}, \mathrm{B}$ & $\mathrm{A}, \mathrm{B}$ & A & $\mathrm{A}, \mathrm{B}$ & $\begin{array}{c}\mathrm{A}, \mathrm{B} \\
\mathrm{B}\end{array}$ & A & B & B & A & $\mathrm{A}, \mathrm{B}$ & $\mathrm{A}, \mathrm{B}$ & \\
\hline & & $B$ & & $B$ & & & & & & B & B & \\
\hline & & A & & A,B & & & A & & & $\mathrm{A}, \mathrm{B}$ & & \\
\hline & A & A & & A & & $\mathrm{A}, \mathrm{B}$ & A & A & A & B & $\mathrm{A}, \mathrm{B}$ & \\
\hline & A & A & A & A & A & A & & A & & A & $\mathrm{A}, \mathrm{B}$ & $\mathrm{A}, \mathrm{B}$ \\
\hline & & & A & & & $\mathrm{A}, \mathrm{B}$ & & A & & B & $\mathrm{A}, \mathrm{B}$ & B \\
\hline & & & & & & B & & A & & B & B & B \\
\hline & & $\mathrm{A}, \mathrm{B}$ & & $\mathrm{A}, \mathrm{B}$ & B & A & A & $\mathrm{A}, \mathrm{B}$ & $\mathrm{A}, \mathrm{B}$ & A & & \\
\hline
\end{tabular}

Táxons

Durinskia baltica (Levander) Carly \& Cox

Gymnodinium sp.

cf. Gymnodinium

Katodinium sp.

Peridinum willei Huitfeld-Kaas

Peridinum cf. lomnickii Wolozynska

Peridinum gatunense Nygaard

Peridinum umbonatum Stein

cf. Peridinum

Thompson intermedium (Thompson)

Bourrelly

Woloszynskia neglecta (Scilling) Thompson

A quantificação dos organismos foi efetuada segundo método de Utermöhl (1958), em câmaras de sedimentação e microscópio invertido (Olympus IX70) somente para amostras coletadas em zonas pelágicas. $\mathrm{O}$ volume de material analisado variou de $1 \mathrm{~mL}$ a $32 \mathrm{~mL}$, para atingir uma eficiência amostral mínima de $80 \%$ (Papas \& Stoermer 1996). A densidade foi estimada em cél.L $L^{-1}$, de acordo com APHA (1992). Adotou-se o critério proposto por Lobo \& Leighton (1986) para abundância e dominância das espécies em cada amostra. A riqueza de espécies foi verificada pelo número de táxons presentes em cada amostra e a diversidade específica pelo índice de Shannon-Wiener (Shannon \& Weaver 1949 apud Krebs 1978).

O tratamento estatístico dos dados foi efetuado somente para amostras quantificadas (Tab. 2) através de análise descritiva, ANOVA bi-fatorial, análise de correlação $(r$-Pearson, p<0,05) e análise de agrupamento (técnica de ligação simples e distância euclidiana). Dados de densidade das espécies foram transformados $(\log x+1)$ antes de processar as análises. Análise descritiva foi utilizada para verificar a distribuição dos dados de riqueza, diversidade e densidade em relação aos tipos de habitats, áreas estudadas e hidroperíodos. ANOVA foi empregada para verificar a significância da variação da distribuição dos dados usados na análise descritiva, bem como a correlação entre os mesmos. Análise de agrupamento foi utilizada para verificar a similaridade entre as unidades amostrais (habitats e hidroperíodos) em relação aos dados de densidade das espécies. Análise de espécie indicadora (Dufrêne \& Legendre 1997) foi realizada com o intuito de determinar as espécies que mostraram alta especificidade de habitat.

Análise descritiva, de variância (ANOVA) e de correlação $r$-Pearson $(\mathrm{p}<0,05)$ foram realizadas com o programa Statistica ${ }^{\circledR}$ (Statsoft Inc. 1996), enquanto que, análises de agrupamento (técnica de ligação simples e distância euclidiana) e de espécie indicadora foram efetuadas com o programa PC-ORD ${ }^{\circledR}$ (MacCune \& Mefford 1995).

\section{Resultados}

Riqueza - A riqueza de dinoflagelados nas áreas de estudo foi baixa, um total de 11 táxons foram registrados (Tab. 1; Fig. 2-18). Em águas continentais dulceaquícolas geralmente esse grupo apresenta baixa 


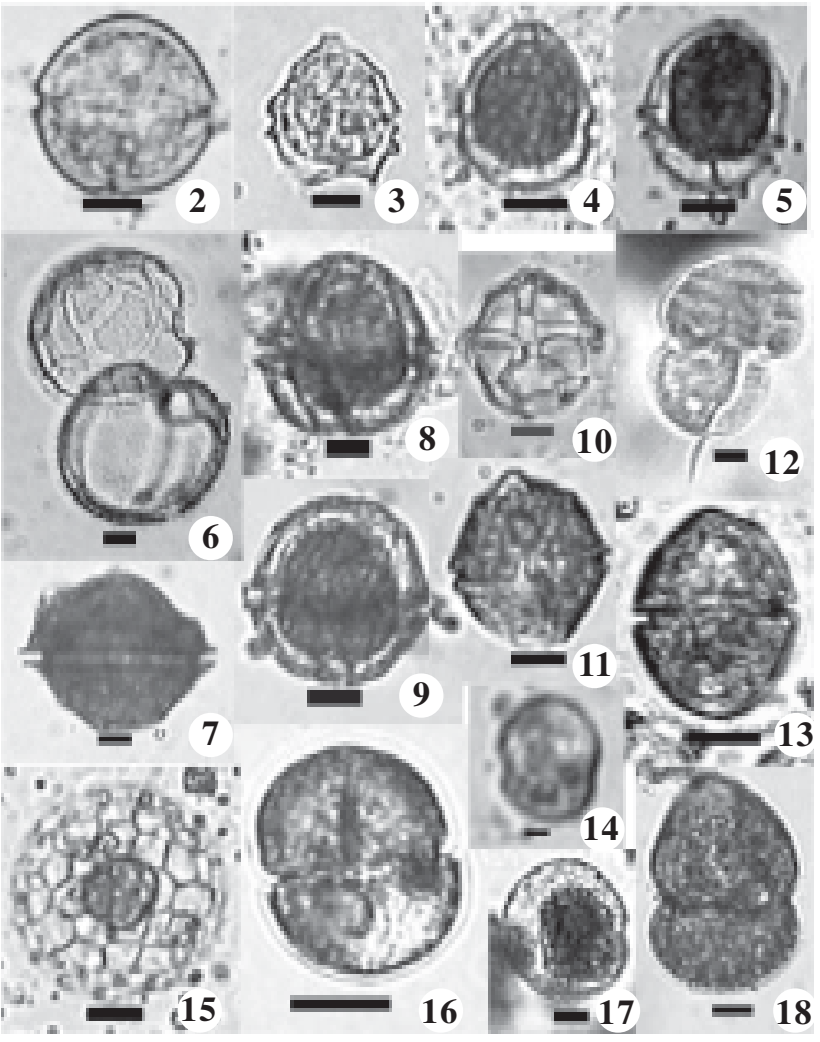

Figura 2-18. Dinophyceae registradas para os habitats associados às áreas da Lagoa do Casamento e do Butiazal de Tapes: 2. Durinskia baltica (Levander) Carty \& Cox. 3. Peridinium umbonatum Stein. 4-5. Peridinium cf. lomnickii Woloszynska. 6-7. Peridinium gatunense Nygaard. 8-9. Peridinium willei HuitfeldKaas. 10-11. Thompsodinium intermedium (Thompson) Bourelly. 12. Katodinium sp. 13. cf. Peridinium. 14. cf. Gymnodinium. 15-17. Woloszynskia neglecta (Schilling) Thompson. 18. Gymnodinium sp. $($ Escala $=10 \mu \mathrm{m}$ exceto $13=2,5 \mu \mathrm{m})$.

riqueza, quando comparado com outros grupos de algas, associado ao fato que algumas espécies são bastante frágeis, sendo eliminadas pela fixação.

A riqueza nas amostragens qualitativas (zonas pelágica e litorânea) variou em relação aos pontos de amostragem (Fig. 19; Tab. 1). As lagoas fechadas na área do Butiazal de Tapes (P13 e P14 - Lagoa do Charutão, P16 e P17 - Lagoa das Capivaras, P19 e P20 - lagoa e banhado entre dunas), bem como o canal do Sangradouro (P10) e banhado da Fazenda Rincão do Anastácio (P5 e P6), na área da Lagoa do Casamento, apresentaram maior riqueza de táxons (6 a 8 spp.) em comparação com as lagoas abertas em conexão com a laguna dos Patos (1 a 4 spp.). A riqueza nestas últimas (P3, P4, P7 a P9B, P11 a P12B) esteve relacionada com o hidroperíodo, observando-se maior número de táxons no período de águas altas (Fig. 19) devido à contribuição de águas oriundas da bacia do lago Guaíba.
Com relação a análise quantitativa (Tab. 2), os resultados da ANOVA demonstraram que o tipo de habitat influenciou significativamente $(\mathrm{p}<0,01)$ a riqueza, onde a média mais elevada ocorreu no canal (quatro espécies) e a menor nas lagoas abertas (uma espécie). Esta variação pode ser bem observada (Fig. 20A) no gráfico de análise descritiva dos dados, onde as lagoas fechadas e o canal do Sangradouro foram os habitats mais ricos em dinoflagelados.

Densidade - A densidade total de dinoflagelados variou de 0 a 6512 cél $\mathrm{L}^{-1}$, apresentando valores superiores no período de águas baixas (Tab. 2, Fig. 20E). O valor máximo (6389 cél $\left.\mathrm{L}^{-1}\right)$ foi observado no canal do Sangradouro (P10), devido a dominância de Durinskia baltica (Levander) Carty \& Cox. Densidades relativamente altas $\left(>58\right.$ cél $\left.\mathrm{L}^{-1}\right)$, encontradas nas lagoas fechadas (P14, P17 e P21) da área do Butiazal de Tapes, foi decorrente da abundância de Peridinium gatunense Nygaard, no período de águas altas, e Peridinium umbonatum Stein, no período de águas baixas. A distribuição e abundância dos mesmos foi tratada anteriormente por Cardoso e Torgan (2005). A densidade elevada ( 213 cél L $^{-1}$ ), encontrada no período de águas baixas na Lagoa das Capivaras (P17), foi decorrente da dominância de Woloszynskia neglecta (Schilling) Thompson. As baixas densidades (0 a 10 cél $\mathrm{L}^{-1}$ ) ocorreram em todos os pontos de amostragem na área da Lagoa do Casamento, exceto para o canal do Sangradouro (P10) e Lagoa dos Gateados Sul (P12A e B) no período de águas baixas.

Os resultados da ANOVA demonstraram que a densidade de dinoflagelados foi significativamente $(\mathrm{p}<0,01)$ influenciada pelo hidroperíodo (média mais elevada no período de águas baixas); pelo tipo de habitat (canal Sangradouro como o local com maior densidade média); e espaço-temporal pela interação entre tipo de habitat com hidroperíodo. Estas variâncias podem ser bem observadas nos gráficos de análise descritiva dos dados (Fig. 20E, 20B, 20F, respectivamente).

Durante o período de águas baixas ocorreu correlação significativa $(r=1,00 \mathrm{p}<0,05)$ entre os habitats conectados à Lagoa do Casamento (P12, P10 e P4). Este fato esteve relacionado com a dominância de Durinskia baltica nestes pontos de amostragem (Tab. 2). Contudo, no período de águas altas, correlação significativa foi verificada entre os ecossistemas associados à Lagoa do Casamento $(\mathrm{r}=0,82$ a 1,00 $\mathrm{p}<0,05$ nos P9, P10, P4 e P3), devido à dominância de Durinskia baltica e/ou de Peridinium gatunense, e 
Tabela 2. Densidade (cél L-1) e diversidade (bits ind ${ }^{-1}$ ) dos táxons de Dinophyceae e variáveis abióticas nos habitats aquáticos dos ecossistemas associados à Lagoa do Casamento e ao Butiazal de Tapes, durante o hidroperíodo de águas altas e baixas. $($ SD = sem dados).

\begin{tabular}{|c|c|c|c|c|c|c|c|c|c|c|}
\hline \multirow{3}{*}{$\begin{array}{l}\text { Hidroperíodo } \\
\text { Áreas } \\
\text { Pontos de amostragem }\end{array}$} & \multicolumn{10}{|c|}{ Águas Altas } \\
\hline & \multicolumn{7}{|c|}{ Lagoa do Casamento } & \multicolumn{3}{|c|}{ Butiazal de Tapes } \\
\hline & P3 & P4 & P9A & P9B & P10 & $\mathrm{P} 12 \mathrm{~A}$ & P12B & P14 & P17 & P21 \\
\hline Durinskia baltica (Levander) Carty \& Cox & 0,5 & 3,70 & 0,20 & & 9,30 & & & & & \\
\hline Peridinium cf. lomnickii Woloszynska & & & & & 0,10 & & & 10,10 & 6,10 & \\
\hline Peridinium gatunense Nygaard & & 4,20 & 0,10 & & 0,20 & 0,1 & & 98,10 & 18,90 & 58,0 \\
\hline Peridinium umbonatum Stein & & & & & & & & 28,80 & 1,90 & \\
\hline Peridinium willei Huitfeld-Kaas & & & & & 0,10 & & & & & \\
\hline \multicolumn{11}{|l|}{$\begin{array}{l}\text { Thompsodinium intermedium (Thompson) } \\
\text { Bourrelly }\end{array}$} \\
\hline Woloszynskia neglecta (Schilling) Thompson & & & & & 0,50 & & & 0,10 & 0,40 & \\
\hline Densidade total & 0,5 & 7,90 & 0,30 & & 10,10 & 0,1 & & 137,20 & 27,30 & 58,0 \\
\hline Diversidade & 0,0 & 0,69 & 0,56 & & 0,36 & 0,0 & & 0,79 & 0,84 & 0,0 \\
\hline \multicolumn{11}{|l|}{ Variáveis abióticas } \\
\hline temperatura $\mathrm{H}_{2} \mathrm{O}$ & 20,6 & 15,90 & 16,60 & 16,6 & 16,20 & 17,0 & 17,20 & 16,90 & 16,00 & 17,0 \\
\hline pH & 6,7 & 8,80 & 6,80 & 6,8 & 6,20 & 6,8 & 6,80 & 6,50 & 6,70 & 6,7 \\
\hline condutividade $\left(\mu \mathrm{S} \mathrm{cm}^{-1}\right)$ & 107,0 & SD & 195,00 & 171,0 & SD & 187,0 & 187,00 & 28,30 & 35,00 & 25,2 \\
\hline transparência Secchi $(\mathrm{cm})$ & 35,0 & 27,50 & 25,00 & 25,0 & 55,00 & 30,0 & 25,00 & 35,00 & 180,00 & 55,0 \\
\hline profundidade coluna d'água $(\mathrm{cm})$ & 270 & SD & 130 & 115 & 280 & 250 & 295 & 115 & 200 & 65 \\
\hline
\end{tabular}

Hidroperíodo

Águas Baixas

\begin{tabular}{|c|c|c|c|c|c|c|c|c|c|c|}
\hline \multirow{2}{*}{$\begin{array}{l}\text { Áreas } \\
\text { Pontos de amostragem }\end{array}$} & \multicolumn{7}{|c|}{ Lagoa do Casamento } & \multicolumn{3}{|c|}{ Butiazal de Tapes } \\
\hline & P3 & P4 & $\mathrm{P} 9 \mathrm{~A}$ & $\mathrm{P} 9 \mathrm{~B}$ & P10 & $\mathrm{P} 12 \mathrm{~A}$ & P12B & P14 & P17 & $\mathrm{P} 21$ \\
\hline $\begin{array}{l}\text { Durinskia baltica (Levander) Carty \& Cox } \\
\text { Peridinium cf. lomnickii Woloszynska }\end{array}$ & & 0,50 & & & 6389,3 & 114,00 & 368,00 & & & \\
\hline P. gatunense Nygaard & & & & & & & & & & 6,00 \\
\hline P. umbonatum Stein & & & & & & & & 102,00 & & 8,00 \\
\hline P. willei Huitfeld-Kaas & & & & & 96,0 & & & & & \\
\hline $\begin{array}{l}\text { Thompsodinium intermedium (Thompson) } \\
\text { Bourrelly }\end{array}$ & & & & & & & & & & \\
\hline Woloszynskia neglecta (Schilling) Thompson & & & & & 26,7 & 3,00 & 3,00 & & 213,30 & \\
\hline Densidade total & & 0,50 & & & 6512,0 & 117,00 & 371,00 & 102,00 & 213,30 & 14,00 \\
\hline Diversidade & & 0,00 & & & 0,1 & 0,12 & 0,05 & 0,50 & 0,00 & 0,88 \\
\hline $\begin{array}{l}\text { Variáveis abióticas } \\
\text { temperatura } \mathrm{H}_{2} \mathrm{O}\end{array}$ & 22,4 & 22,50 & 20,30 & 20,3 & 321,8 & 20,30 & 20,40 & 34,00 & 25,70 & 31,20 \\
\hline $\mathrm{pH}$ & 6,9 & 6,90 & 7,20 & 7,4 & 6,1 & 6,90 & 6,90 & 6,30 & 5,60 & 6,30 \\
\hline condutividade $\left(\mu \mathrm{S} \mathrm{cm}^{-1}\right)$ & 119,0 & 80,00 & 230,00 & 250,0 & 13,7 & 116,50 & 12,60 & 22,10 & 12,00 & 13,30 \\
\hline transparência Secchi (cm) & 25,0 & 30,00 & 12,50 & 12,5 & 50,0 & 25,00 & 25,00 & SD & SD & $\mathrm{SD}$ \\
\hline profundidade coluna d'água (cm) & 200 & 200 & 100 & 60 & 130 & 250 & 275 & SD & SD & SD \\
\hline
\end{tabular}

entre os ecossistemas associados ao Butiazal de Tapes $(\mathrm{r}=0,95$ a $0,96 \mathrm{p}<0,05$ nos P14, P17 e P21), devido a dominância de Peridinium gatunense. A dominância desta última espécie também foi responsável pela alta correlação $(r=0,95$ a 1,00 p < 0,05) entre a Lagoa dos Gateados Sul (P12) com os ecossistemas associados ao Butiazal de Tapes (P14, P17 e P21).
Diversidade - Os valores máximos da diversidade específica da comunidade de dinoflagelados (Tab. 2; Fig. 20C) foram registrados nas lagoas fechadas, como a Lagoa das Capivaras (P17; 0,84 bits.ind ${ }^{-1}$ ), no período de águas altas, e Lagoa Redonda (P21; 0,88 bits ind $\left.^{-1}\right)$, no período de águas baixas. Valores nulos foram registrados nos pontos de amostragem 


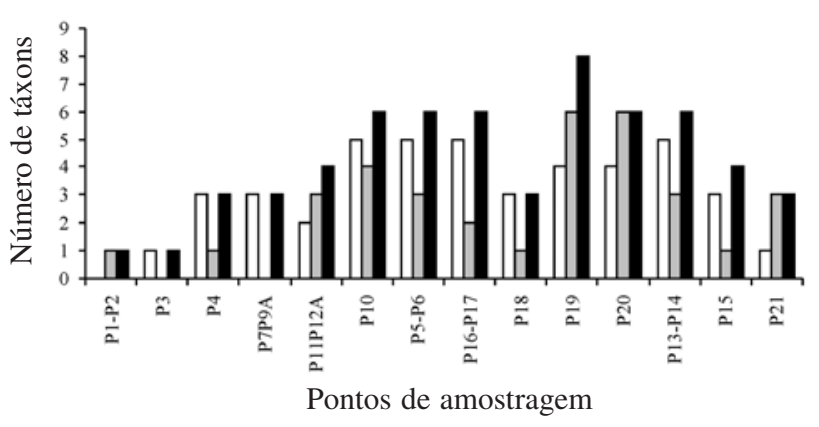

Figura 19. Riqueza de dinoflagelados nos pontos de amostragem para os períodos de águas altas (A) e baixas (B). ( $\square=\mathrm{A}$; $\square=\mathrm{B}$; $\mathbf{u}=$ Total).

onde apenas uma espécie foi registrada, pois a eqüidade torna-se também nula.

Os resultados da ANOVA mostraram que o tipo de habitat e áreas amostradas influenciaram significativamente $(\mathrm{p}<0,01)$ a diversidade. Com relação ao tipo de habitat, a diversidade média foi menor nas lagoas

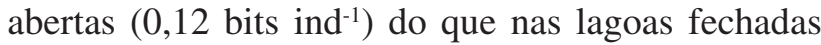

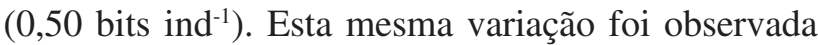
na análise efetuada para as áreas, visto que, o primeiro tipo de habitat ocorre na área da Lagoa do Casamento enquanto que as lagoas fechadas, na área do Butiazal de Tapes. Estas variâncias podem ser bem observadas nos gráficos de análise descritiva dos dados (Fig. 20C-D, respectivamente).

Similaridade - A análise de agrupamento dos táxons, nos pontos de amostragem, evidenciou a ocorrência de dois grupos com 50\% de informação (Fig. 21). O grupo 1 formado pela Lagoa das Capivaras (P17) que diferiu das demais unidades amostrais pela dominância de Woloszynskia neglecta, no período de águas baixas, onde foi registrado o $\mathrm{pH}$ mais baixo $(5,6)$ (Tab. 2). O grupo 2 foi formado pelas demais unidades amostrais, estando estas subdivididas em dois subgrupos. O subgrupo 2a, composto pelas lagoas abertas e canal na área da Lagoa do Casamento, devido a dominância de Durinskia baltica, e onde teve-se o menor registro de temperatura da água $\left(15,9^{\circ} \mathrm{C}\right)$ e maior valor de $\mathrm{pH}(8,8)$ (Tab. 2). O subgrupo $2 \mathrm{~b}$, formado preferencialmente pelas lagoas fechadas da área do Butiazal de Tapes, esteve caracterizado pela dominância de Peridinium gatunense ou $P$. umbonatum, juntamente com os valores mais elevados de temperatura da água $\left(31,2\right.$ e $\left.34^{\circ} \mathrm{C}\right)$ (Tab. 2).

Espécies indicadoras - A análise de espécie indicadora revelou duas espécies capazes de indicar a especificidade de habitat ou hidroperíodo. Peridinium umbonatum foi uma espécie significativa ( $\mathrm{p}<0,05)$, cujo valor indicador foi de 59,4\%, especificando os habitats de lagoas pequenas, rasas, fechadas e isoladas da área do Butiazal de Tapes. Peridinium gatunense foi outra espécie significativa $(\mathrm{p}<0,05)$ cujo valor indicador foi de $63,2 \%$, especificando o período de águas altas.

\section{Discussão}

A comunidade de dinoflagelados, neste estudo, apresentou baixa riqueza (11 táxons) e diversidade

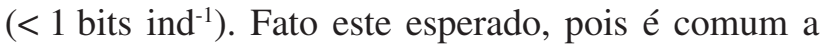
ocorrência de poucas espécies deste grupo em águas dulceaquiícolas. No Estado do Rio de Janeiro, onde um número expressivo de trabalhos sobre a diversidade de algas foi realizado (Menezes \& Teixeira Junior 2001) somente 16 táxons infragenéricos foram registrados. À semelhança de nosso estudo, Peridinium foi o gênero mais bem representado em número de táxons.

Segundo Pollingher (1988), Peridinium é um dos gêneros de dinoflagelados mais bem conhecido, sob o ponto de vista taxonômico e ecológico. Popovsky \& Pfiester (1990) descreveram 20 espécies com suas respectivas variedades. Embora $P$. gatunense e P. umbonatum possuam ampla distribuição geográfica, existia uma lacuna no conhecimento sobre a distribuição e abundância destas duas espécies na região subtropical brasileira. As primeiras informações sobre a variação espacial e temporal destas espécies encontram-se em Cardoso \& Torgan (2005).

$\mathrm{O}$ cálculo de diversidade em comunidades com um número reduzido de táxons não contribuiu para uma interpretação da estrutura da mesma, quando comparada com outras comunidades com maior riqueza de táxons. Uma alta diversidade não representa um alto valor ecológico (Dunn 1994), mostrando-se um critério questionável especialmente quando habitats com diferentes níveis de produtividade são comparados, ou quando o número de espécies raras é grande. Neste caso, as espécies indicadoras oferecem uma maior contribuição sobre as condições ecológicas.

A novidade da análise de espécie indicadora de Dufrêne \& Legendre (1997) é a combinação da abundância relativa de espécies com sua freqüência relativa de ocorrência nos vários grupos de unidades amostrais. Espécies indicadoras são definidas como aquelas mais características de cada grupo, encontradas, principalmente, em um único grupo e presente na maioria das unidades amostrais referentes. 
No presente estudo, duas espécies de Peridinium destacaram-se como indicadoras de habitats e hidroperíodo: $P$. umbonatum em lagoas rasas e fechadas, enquanto que, $P$. gatunense durante o período de águas altas. Estas duas espécies poderão, provavelmente, ser usadas em monitoramentos futuros da conservação de habitats bem como de mudanças climáticas locais.

A análise de agrupamento mostrou que, dentre as seis variáveis abióticas analisadas, $\mathrm{pH}$ e temperatura
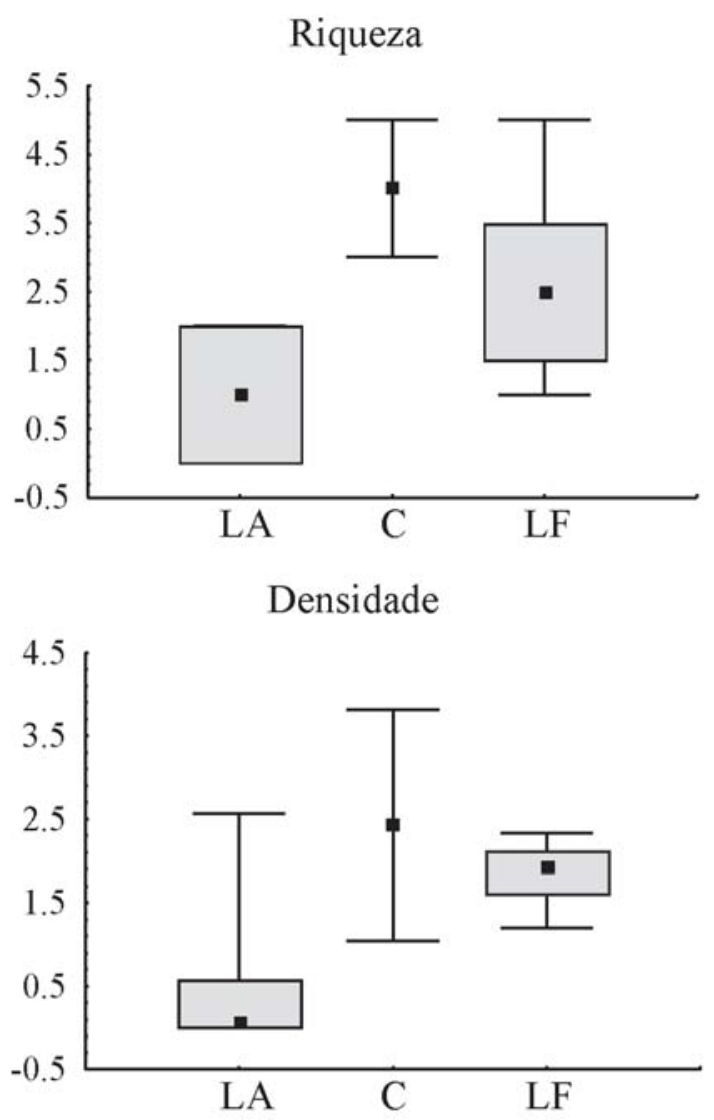

Diversidade

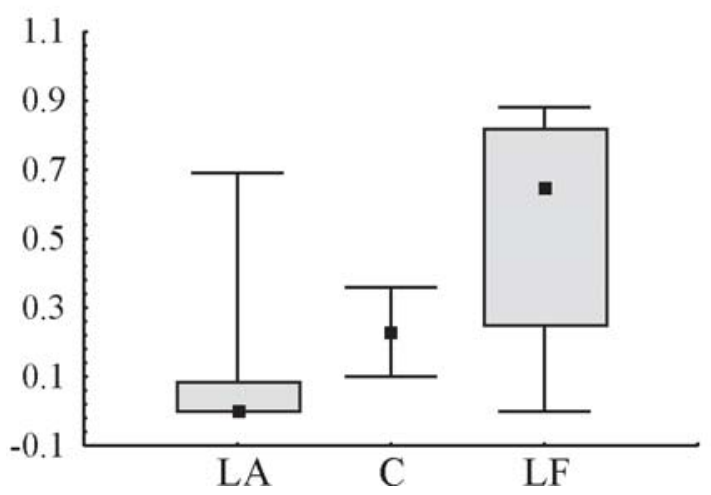

da água foram as que mais influenciaram na estrutura das populações de dinoflagelados neste estudo. A variação do $\mathrm{pH}$ entre os hidroperíodos foi geralmente baixa no mesmo ambiente, exceto para a Lagoa do Casamento (P4) e para a Lagoa das Capivaras (P17). A temperatura da água foi a variável mais uniforme em todos os habitats, com temperaturas mais baixas na estação do outono (período de águas altas) do que na primavera (período de águas baixas). Em um estudo sobre dinoflagelados em 86 corpos de água na Hungria

\section{Diversidade}

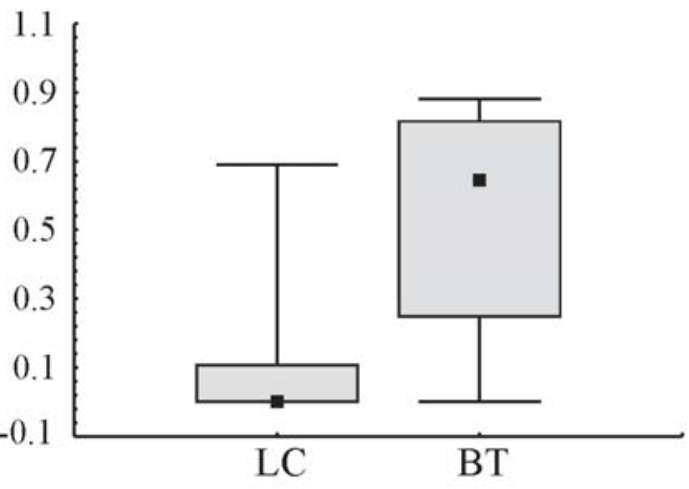

Densidade

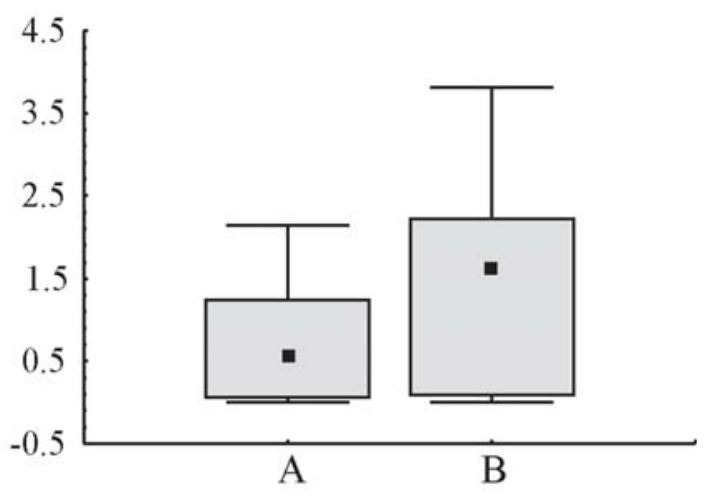

Densidade

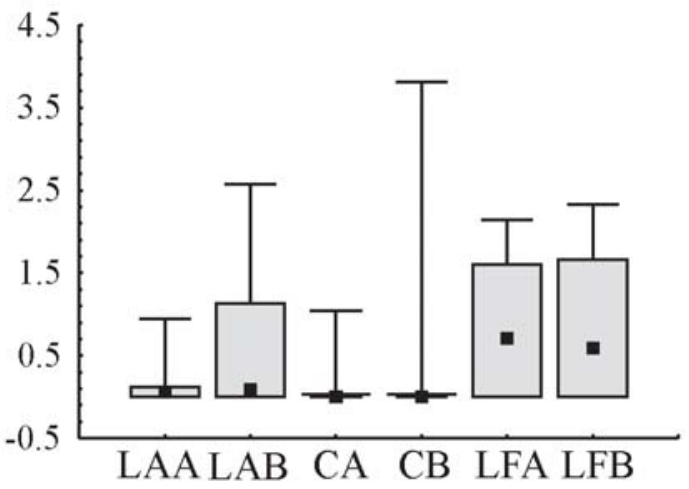

Figura 20. Riqueza (A), densidade (B) e diversidade (C) em relação aos tipos de habitats (LA= lagoas abertas; C= canal; $\mathrm{LF}=$ lagoas fechadas); diversidade (D) nas áreas estudadas (LC= Lagoa Casamento; BT= Butiazal de Tapes); densidade nos hidroperíodos amostrados (E) (A- águas altas e B- águas baixas) e na combinação entre tipos de habitats e hidroperíodos (F). 


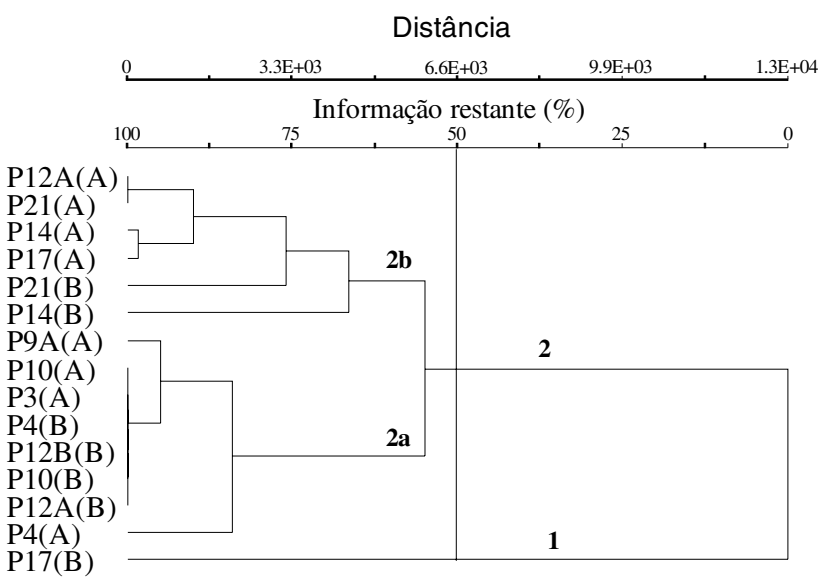

Figura 21. Dendograma dos pontos de amostragem no período de águas altas (A) e águas baixas (B).

foram registrados 23 espécies, onde a análise de agrupamento mostrou cinco grupos de organismos, que tiveram a distribuição ao longo de um gradiente de temperatura (Grigorszky et al. 2003a).

A ocorrência de habitats como lagoas pequenas, rasas e isoladas entre dunas, na área do Butiazal de Tapes, contribuiu para o aumento na riqueza dos dinoflagelados. Estes habitats, não foram influenciados pelo hidroperíodo. Por outro lado, na área da Lagoa do Casamento, a conectividade entre as lagoas pode ser considerado um fator determinante na distribuição mais homogênea da densidade dos dinoflagelados no período de águas altas.

\section{Agradecimentos}

Ao Conselho Nacional de Desenvolvimento Científico e Tecnológico (CNPq), pela concessão de bolsa recém doutor (RD) à primeira autora e bolsa produtividade em pesquisa à segunda autora. Agradecimento especial para Dra. Mariângela Menezes do Museu Nacional do Rio de Janeiro (UFRJ) por disponibilizar sua bibliografia e pela confirmação dos táxons identificados; à direção do Museu de Ciências Naturais da Fundação Zoobotânica do Rio Grande do Sul, pela concessão de infra-estrutura para a execução desta pesquisa, que fez parte do Projeto PROBIO em parceria com o CNPq e Ministério do Meio Ambiente; ao engenheiro José Carlos Martins, Diretor de Hidrovias da Secretaria dos Transportes do Rio Grande do Sul, por fornecer dados de nível d'água da estação localizada na Praça da Harmonia, lago Guaíba.

\section{Referências bibliográficas}

APHA. 1992. Standart Methods for the examination Water and Wastewater. 18 ed. Washington, American Public Health Association.

Cardoso, L.S. \& Torgan, L.C. 2005. Distribuição e densidade de Peridinium gatunense e $P$. umbonatum (Dinophyceae) em ecossistemas da zona costeira no sul do Brasil. Pp. 517-526. In: Anais da X Reunião Brasileira de Ficologia. Salvador 2004. Rio de Janeiro, Museu Nacional. Série Livros, 10.

Dufrêne, M. \& Legendre, P. 1997. Species assemblages and indicator species: the need for a flexible asymmetrical approach. Ecological Monographs 67(3): 345-366.

Dunn, C. 1994. Gaps in GAP. Plant Science Bulletin 40: 120-121.

Franceschini, I.M. 1992. Algues d'eau douce de Porto Alegre, Brésil (lês Diatomophycées exclues). Berlin, J. Cramer. (Bibliotheca Phycologica, 92).

Garcia, M. \& Vélez, E. 1995. Algas planctônicas da Lagoa Emboaba, Planície Costeira do Rio Grande do Sul: Avaliação qualitativa. Boletim do Instituto de Biociências da Universidade Federal do Rio Grande do Sul 54: 75-114.

Grigorszky, I.; Borics, G.; Padisák, J.; Tótmérész, B.; Vasas, G.; Nagy, S. \& Borbély, G. 2003a. Factors controlling the occurrence of Dinophyta species in Hungary. Hydrobiologia 506-509: 203-207.

Grigorszky, I.; Krienitz, L.; Padisák, J.; Borics, G. \& Vasas, G. 2003b. Redefinition of Peridinium lomnickii Woloszynska (Dinophyta) by scanning electronmicroscopical survey. Hydrobiologia 502: 349-355.

Krebs, C.J. 1978. Ecology: the experimental analysis of distribution and abundance. 2 ed. New York, Harper \& Row.

Lobo, E.A. \& Leighton, G. 1986. Estructuras comunitarias de las fitocenosis planctónicas de los sistemas de desembocaduras de rios y esteros de la zona central de Chile. Revista de Biologia Marina 22(1): 1-29.

Maccune, B. \& Mefford, M.J. 1995. PC-ORD. Multivariate analysis of ecological data, version 2.0. Oregon, MJM Software Design gleneden Blach.

Menezes, M. \& Teixeira Junior, E.C. 2001. Dinophyta. pp. 105-109. In: M. Menezes \& I.C.A. Dias (orgs.). Biodiversidade de Algas de Ambientes Continentais do Estado do Rio de Janeiro. v.9, Rio de Janeiro, Série Livros, Museu Nacional.

Pappas, J.L. \& Stoermer, E.F. 1996. Quantitative method for determining a representative algal sample count. Journal of Phycology 32: 693-696.

Pollingher, U. 1986. Phytoplankton periodicity in a subtropical lake (Lake Kinneret, Israel). Hydrobiologia 138: $127-138$.

Pollingher, U. 1988. Freshwater armored dinoflagellates: growth, reproduction strategies, and population dynamics. Pp. 134-174. In: C.D. Sandgren (ed.). Growth and Reproductive Strategies of Freshwater Phytoplankton. Cambridge, Cambridge University. 
Popovsky, J. 1970. Some thecate dinoflagellates from Cuba. Archiv fur Protistenkunde 112(Suppl.): 252-258.

Popovsky, J. 1983. Problems in the determination of freshwater dinoflagellates (Dinophyceae). Schweizerische Zeitschrift Fur Hydrologie 45 (2): 365-372.

Popovsky, J. \& Pfiester, L.A. 1986. A taxonomical note to the section umbonatum of the genus Peridinium Ehrenberg, 1932 (Dinophyceae). Archiv fur Protistenkunde 132: 73-77.

Popovsky, J. \& Pfiester, L.A. 1990. Dinophyceae (Dinoflagellida). Pp. 1-272. In: H. Ettl; J. Gerloff; H. Heynig \& D. Mollenhauer (eds.). Sübwasserflora von Mitteleuropa. 6. Sttugart, Gustav Fischer Verlag.

Rocha, O. 2000. Perfil do Conhecimento de Biodiversidade em Águas Doces no Brasil. São Carlos, COBIO/MMA - GTB/CNPq - NEPAM/UNICAMP, relatório final da Avaliação do estado do conhecimento da diversidade biológica do Brasil.
Rosa, Z.M.; Ungaretti, I.; Kremer, L.M.; Silva, S.M.A.; Callegaro, V.L.M. \& Werner, V.R. 1987. Ficoflora de ambientes lênticos - Estudo preliminar da região de Charqueadas, Rio Grande do Sul, Brasil, com vistas à avaliação ambiental. Acta Botanica Brasilica 1(2): 165-188.

Taylor, F.J.R. 1987. Dinoflagellate morphology. Pp. 24-91. In: F.J.R. Taylor (ed.). The Biology of Dinoflagellates. Oxford, Blackwell scientific Publications

Thompson, R.H. 1950. New genus and new records of freshwater Pyrrophyta in the Desmokontae and Dinophyceae. Lloydia 13(4): 277-299.

Torgan, L.C.; Barreda, K.A. \& Santos, C.B. 2003. Catálogo das algas Cryptophyta, Heterokontophyta, Dinophyta, Haptophyta e Rhodophyta de águas continentais e costeiras do Rio Grande do Sul, Brasil. Iheringia, Ser. Bot., 58(2): 227-250.

Utermöhl, U. 1958. Zur Vervollkommnung der quantitativen Phytoplankton-Methodik. Mitteilungen der Internationalen Vereinigung für theoretische und angewandte Limnologie 19: 1-38. 